\title{
C-band general Class-J power amplifier using GaN HEMT
}

\author{
Zhebin $\mathrm{Hu}^{\text {a)}}$, Chaoyi Huang, Songbai He, Fei You, \\ Shuyi Xie, and Haodong Lin \\ School of Electronic Engineering, University of Electronic Science and \\ Technology of China, Chengdu, 611731, China \\ a)huzhebin.ee@gmail.com
}

Abstract: In this paper, a $C$-band wideband power amplifier (PA) using a GaN HEMT is proposed. The general Class-J mode, which offer a bigger design space for the fundamental and harmonic terminations, is introduced to implement wideband PA. Then, to verify the mechanism mentioned above, a wideband PA operating from 3.5 to $6.0 \mathrm{GHz}$ is proposed and fabricated. Meanwhile, the load/source harmonic and baseband impedances are also considered for the linearity. The amplifier exhibits $8-11 \mathrm{~dB}$ gain and $51 \%-64 \%$ drain efficiency (DE) covering from 3.5 to $5.5 \mathrm{GHz}$.

Keywords: $C$-band, Class-J, wideband, power amplifier, linearity optimization

Classification: Microwave and millimeter-wave devices, circuits, and modules

\section{References}

[1] S. C. Cripps, et al:: "On the Continuity of High Efficiency Modes in Linear RF Power Amplifiers," IEEE Microw. Wireless Compon. Lett. 19 (2009) 665 (DOI: 10.1109/LMWC.2009.2029754).

[2] P. Wright, et al.: "A Methodology for Realizing High Efficiency Class-J in a Linear and Broadband PA," IEEE Trans. Microw. Theory Techn. 57 (2009) 3196 (DOI: 10.1109/TMTT.2009.2033295).

[3] J. Moon, et al:: "Investigation of a Class-J Power Amplifier With a Nonlinear Cout for Optimized Operation,” IEEE Trans. Microw. Theory Techn. 58 (2010) 2800 (DOI: 10.1109/TMTT.2010.2077970).

[4] V. Carrubba, et al.: "The Continuous Class-F Mode Power Amplifier," Microwave Integrated Circuits Conference (EuMIC), 2010 European, Paris, 2010, pp. 432-435.

[5] J. Chen, et al.: "Design of Broadband High-Efficiency Power Amplifiers Based on a Series of Continuous Modes," IEEE Microw. Wireless Compon. Lett. 24 (2014) 631 (DOI: 10.1109/LMWC.2014.2331457).

[6] C. Friesicke, et al:: "The Resistive-Reactive Class-J Power Amplifier Mode," IEEE Microw. Wireless Compon. Lett. 25 (2015) 666 (DOI: 10.1109/LMWC. 2015.2463211).

[7] N. B. Carvalho and J. C. Pedro: "Two-tone IMD asymmetry in microwave power amplifiers," IEEE Int. Microw. Symp. (2000) 445 (DOI: 10.1109/ MWSYM.2000.861062).

[8] N. T. Kim: "Design of impedance-matching networks for microwave and 
millimeter-wave amplifier applications," Microw. Opt. Technol. Lett. 27 (2000) 109 (DOI: 10.1002/1098-2760(20001020)27:2<109::AID-MOP8>3.0.CO; 2-0).

[9] P. J. Tasker and J. Benedikt: "Waveform Inspired Models and the Harmonic Balance Emulator,” IEEE Microw. Mag. 12 (2011) 38 (DOI: 10.1109/MMM. 2010.940101).

[10] Y. Ma, et al.: "An open-loop digital predistorter based on memory polynomial inverses for linearization of RF power amplifier," Int. J. RF Micow. Comput.-Aided Eng. 21 (2011) 589 (DOI: 10.1002/mmce.20546).

[11] Y. Ma, et al.: "An Algorithm for Obtaining the Inverse for a Given Polynomial in Baseband," IEICE Trans. Fundam. Electron. Commun. Comput. Sci. 96 (2013) 675 (DOI: 10.1587/transfun.E96.A.675).

\section{Introduction}

To meet the ever-increasing requirements of the high data-rates from consumer market, the wireless high speed data transfer system is highly demanded, especially the wideband $C$-band application. As one of the essential key component in the system, wideband power amplifier (PA) with high efficiency and high linearity is dramatically developed. Class-B and Class-F PAs, which can all offer high efficiency, are not suitable for wideband applications because of the strict harmonic load requirement. To overcome the restriction, continuous mode PAs, known as Class-J [1, 2, 3], continuous Class-F [4], has been proposed. These PA modes can theoretically maintain high efficiency over a continuous range of fundamental and harmonic impedances on the current-generator plane (I-gen plane). Class-J mode is developed from Class-B with additional reactive fundamental and second harmonic impedances. It offers more flexibility in highly efficient PA design. However, the matching network, combined with the parasitics, can not keep the real part of fundamental impedance constant over a desired bandwidth. Therefore, other PA modes $[5,6]$ based on Class-J or continuous Class-F are proposed to extend the design space of fundamental and second harmonics, and also the bandwidth.

In this paper, the general Class-J, which can offer a greater design space for the fundamental and harmonics while keeping the efficiency above a certain value, is introduced for a wideband PA design. Furthermore, a wideband PA operating at $C$-band for wireless high speed data-transfer system is proposed. Besides, the harmonic and baseband impedances of load matching network are optimized for linearity.

\section{General Class-J power amplifier}

The Class-J evolves from the standard Class-B, which has the current like (normalized to the fundamental coefficient)

$$
i(\theta)=\frac{2}{\pi}+\cos \theta+\frac{4}{3 \pi} \cos 2 \theta+\cdots,
$$

where $\theta=2 \pi f t, f$ is the fundamental frequency. And this rectified sinusoid current waveform is assumed for all analysis here. The Class-B exhibits $78.5 \%$ of efficiency with the shorted harmonic loading condition. The voltage waveforms at I-gen plane of Class-J mode is defined as (shown in Fig. 1(a)): 


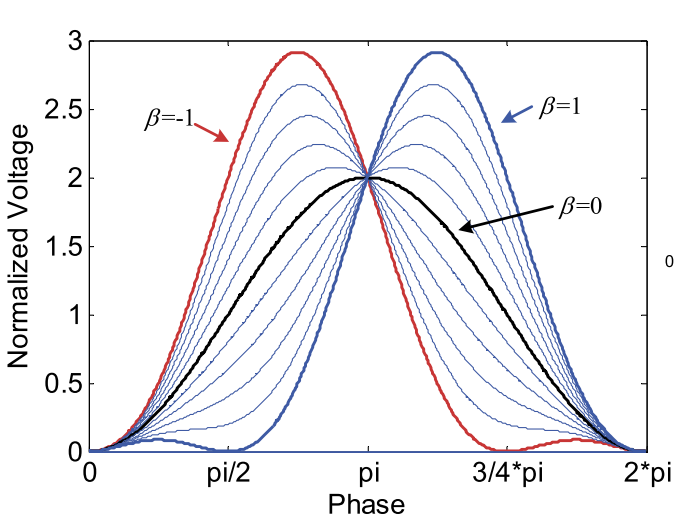

(a)

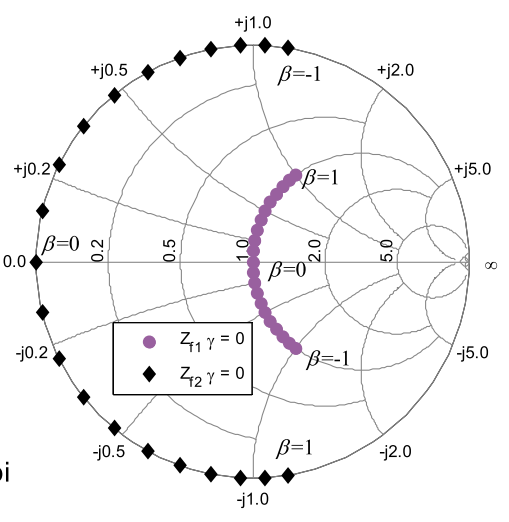

(b)

Fig. 1. (a) voltage waveform at I-gen plane and (b) load condition of Class-J power amplifier.

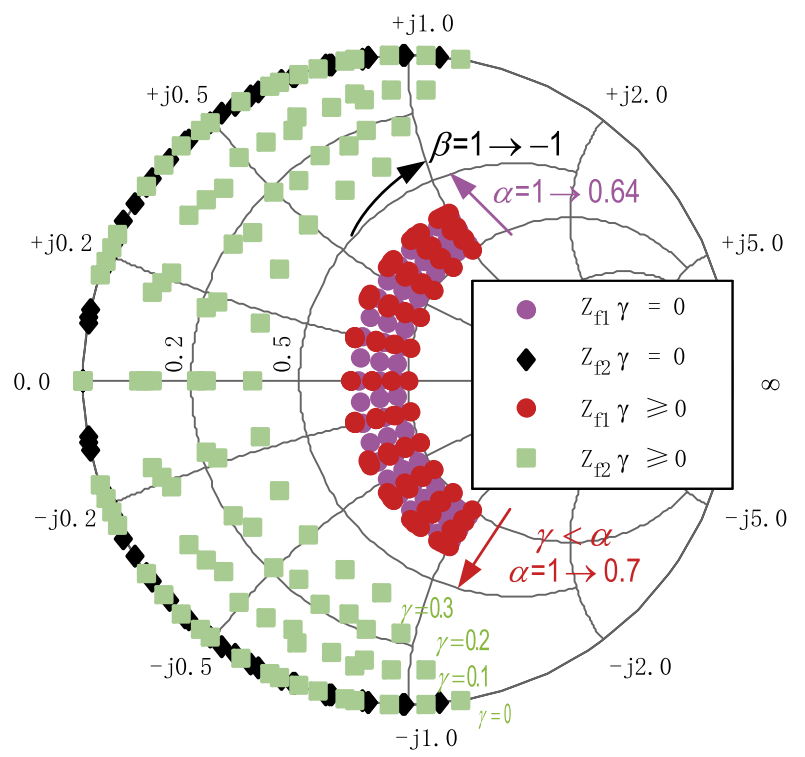

Fig. 2. Impedance design space of the general Class-J mode for $\gamma=0$ or $\gamma \geq 0$ when varying $\alpha, \beta$ in steps of 0.1 . (The theoretical efficiency is above $50 \%$ with $3 \mathrm{~dB}$ output power variance)

$$
v(\theta)=(1-\cos \theta)(1-\beta \sin \theta) .
$$

The first bracket of (2) is the standard voltage waveform of Class-B, while the second bracket is added for the wider design space. Varying $\beta$ from -1 to 1 , the fundamental and second harmonic impedance vary on the constant resistance circle (shown in Fig. 1(b)), and the output power and efficiency keep the same value with Class-B. However, since the load impedance of matching network rotates clockwise in Smith chart, it is not possible to matching all the points on the constant resistance circle. As such, the general Class-J mode is introduced with the equation:

$$
v(\theta)=(1-\alpha \cos \theta)(1-\beta \sin \theta)(1+\gamma \cos \theta) .
$$

The voltage waveform should be kept away from zero crossing and negative voltage and the equivalent loading should be passive (i.e. $0<\alpha \leq 1,-1 \leq \beta \leq 1$ and $0 \leq \gamma<\alpha \leq 1$ ). Then, the load impedance, output power (Pout) and the drain 
efficiency (DE) (all are normalized to the coefficients of Class-B mode) can be yield from (3)

$$
\begin{gathered}
P_{\text {out }}=\alpha-\gamma, \\
\eta=\frac{\alpha-\gamma}{1-\frac{1}{2} \alpha \gamma} * \frac{\pi}{4}, \\
Z_{f 1}=(\alpha-\gamma)+j *\left(-\frac{1}{4} \alpha \beta \gamma+\beta\right), \\
Z_{f 2}=\left(\left(\frac{1}{2} \alpha \gamma\right)+j *\left(\frac{1}{2} \beta \gamma-\frac{1}{2} \alpha \beta\right)\right) * \frac{3 \pi}{4},
\end{gathered}
$$

where $Z_{f 1}, Z_{f 2}$ represent the fundamental and second impedances on the I-gen plane. From (6) and (7), it is obvious that the general Class-J bring the variable resistance of fundamental and second harmonic. Therefore, varying the parameters $\alpha, \beta$ and $\gamma$, it is possible to get more solutions to achieve the desired Pout and efficiency. Fig. 2 shows the general Class-J impedances range for the first two harmonic impedances when the efficiency is above $50 \%$ with $3 \mathrm{~dB}$ output power variance. For $\gamma=0$, there is a large space for the fundamental to obtain the desired performance when the second harmonic loading is purely reactive. In this case, for a wideband output matching network (include the parasitic of the package), the load impedance need to change rapidly to meet the high reflection circle. With non-zero value of $\gamma$, the general Class-J could furtherly alleviate the impedance requirement.

\section{Implementation and experimental results}

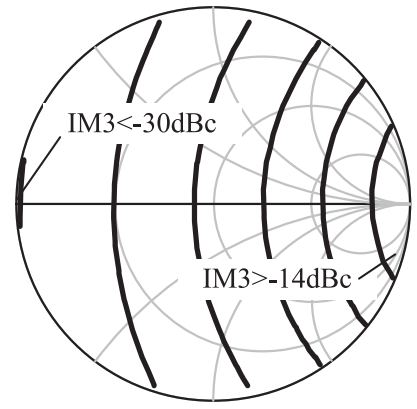

(a) Baseband load-pull results

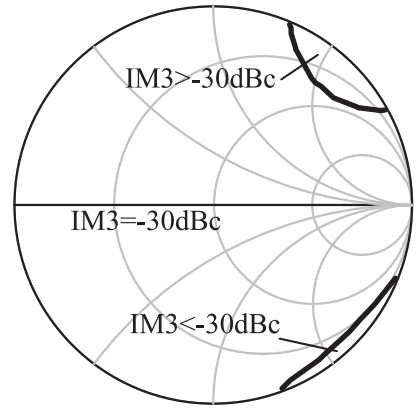

(b) Second harmonic loadpull results

Fig. 3. IM 3 contours of simulated baseband and harmonic load-pull at $5 \mathrm{GHz}$

To valid the theoretical design space in the previous section, the transistor Wolfspeed GaN HEMT CGH40006P is utilized to implement the wideband PA operating from 3.5 to $6.0 \mathrm{GHz}$. Considering the nonlinear parasitic components in the transistor, the practical implementation of precise target load impedance at the I-gen plane face some complications. Moreover, Fig. 2 shows that it is possible to obtain high performance over a wide bandwidth without perfect matching. As such, the PA design mainly focus on the fundamental matching, while keeping the harmonic 


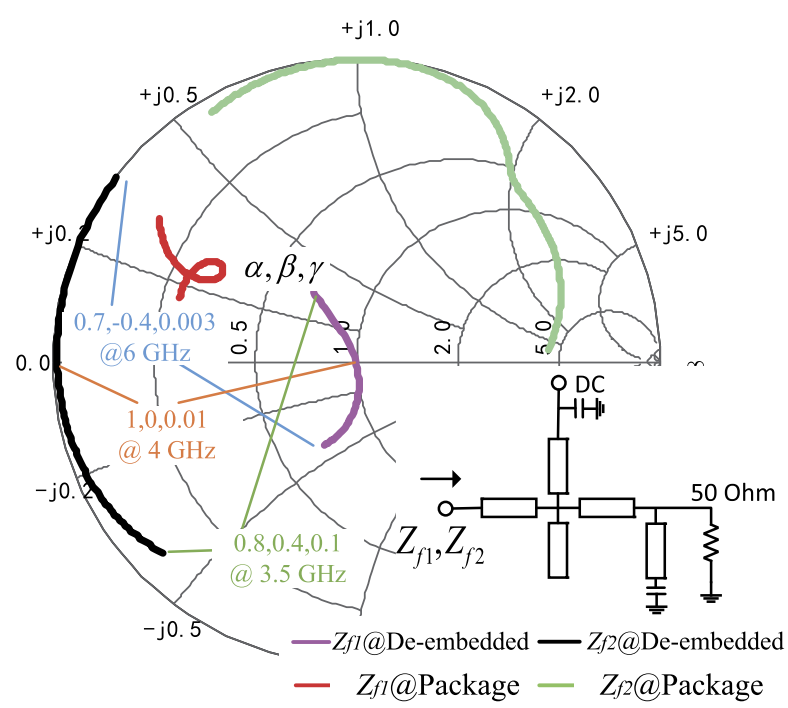

Fig. 4. The output matching network schematic and the impedance trajectories from 3.5 to $6 \mathrm{GHz}$ on I-gen (de-embed the package) and Package plane.

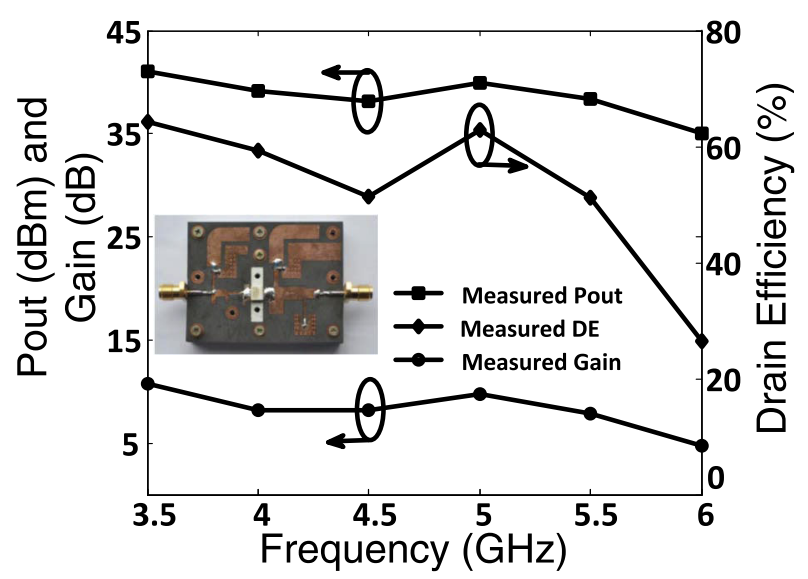

Fig. 5. Fabricated PA and CW Measurement results.

loading in the high efficiency regions. Meanwhile, the linearity of the PA is also considered here. Based on the conclusion in [7], the output baseband and second harmonic impedance are critical to the performance of the linearity. Then, a twotone load pull has been applied at the baseband and second harmonic. As illustrated in Fig. 3, it is found that the IM3 is mainly depended on the output baseband impedance, and thus the baseband load is chosen closely to short. Consequently, the DC-Feed line is placed as close as possible to the transistor. Fig. 4 shows the wideband output matching network [8] used for this design and the impedance trajectories in the Smith chart. The parasitic components are de-embedded based on the approximated network [9] of the CGH40006P. It can be seen that the fundamental and the second harmonic impedances are mostly in the region shown in Fig. 2.

To the end, a PA operated from 3.5 to $6 \mathrm{GHz}$ is fabricated as shown in Fig. 5. It is biased with the drain voltage of $28 \mathrm{~V}$ with a quiescent current of $100 \mathrm{~mA}$. Fig. 5 shows the continuous wave $(\mathrm{CW})$ measurement results from 3.5 to $6.0 \mathrm{GHz}$ with the input power of $30 \mathrm{dBm}$. The measured DE over $50 \%$, output power of 


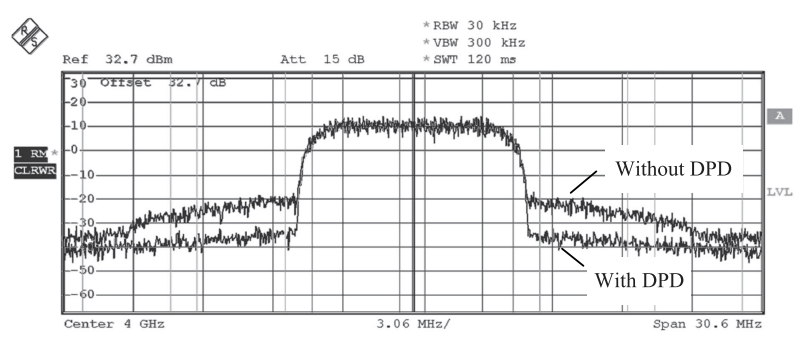

(a) output spectrum(with and w/o DPD)

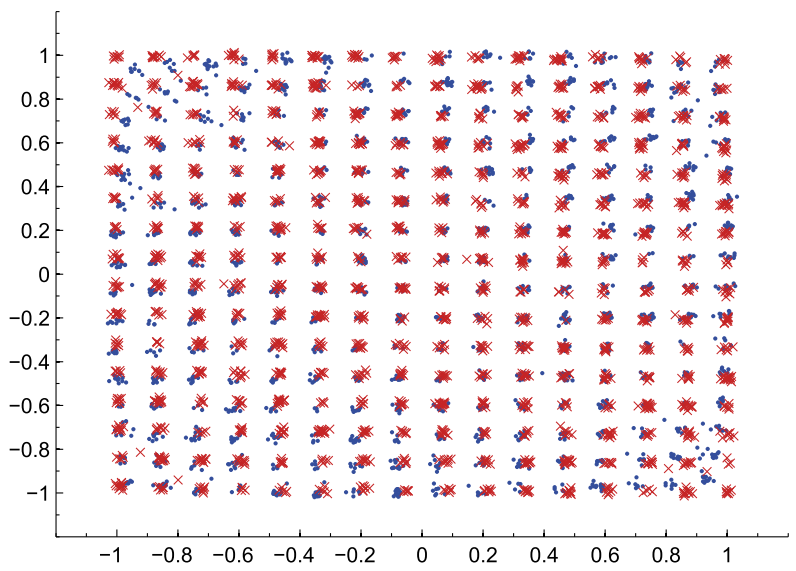

(b) measured constellation(red star for DPD, blue dot for w/o DPD )

Fig. 6. Modulated signal testing at $4 \mathrm{GHz}$

$38-41 \mathrm{dBm}$ and gain of 8-11 dB are achieved in the band between 3.5-5.5 GHz. In addition, to evaluate the performance for practical communication system, the proposed wideband PA is stimulated using a $10 \mathrm{MHz} 256$ QAM modulated signal with PAPR of $6 \mathrm{~dB}$ (shown in Fig. 6). The PA can deliver an average power of $33 \mathrm{dBm}$ with DE over $39 \%$, ACLR of $-45 \mathrm{dBc}$ and EVM of $2 \%$ at $4 \mathrm{GHz}$ using open loop digital pre-distortion (DPD) $[10,11]$.

\section{Conclusion}

In this paper, a general Class-J PA is introduced for wideband PA design. Meanwhile, the effect of harmonic tuning is also considered to improve the linearity. To verify the principle and mechanism, a wideband PA operating from 3.5 to $6 \mathrm{GHz}$ is implemented. Refer to the measurement results, the proposed approach for wideband PA design can be attractive for practical C-band wireless communication.

\section{Acknowledgments}

This work was supported by the National Natural Science Foundation of China under Grant 61271036 and 61571080. 